\title{
RESILIENCIA Y DEPRESIÓN EN ESTUDIANTES DE SECUNDARIA DE LIMA METROPOLITANA CON Y SIN PARTICIPACIÓN EN ACTOS VIOLENTOS
}

\author{
Carlos Velásquez C. ${ }^{1}$, William Montgomery U. \\ Universidad Nacional Mayor de San Marcos, Lima, Perú \\ (RECIBIDO EL 13/09/2009, ACEPTADO EL 30/11/ 2009)
}

\begin{abstract}
RESUMEN
Se realizó un estudio correlacional entre resiliencia y depresión en instituciones educativas de Lima, con alumnos del 3ro-5to de secundaria, de género masculino y femenino, de los cuales una parte no ha participado en actos violentos y otra sí. Los instrumentos utilizados para la evaluación fueron la Escala de Resiliencia y el Inventario de Depresión en la Adolescencia. Los resultados muestran que existen correlaciones positivas en los componentes internos de ambas pruebas, mientras que al correlacionarlas entre ellas la relación es más diversa. Se identificaron diferencias entre los alumnos violentos y no violentos en cuanto a los factores de confianza, autoritarismo e ideas suicidas. Asimismo, en la comparación intersexo, se identificaron diferencias entre mujeres y varones respecto al grado de control emocional, toma de decisiones, autocrítica, propensión al llanto y otras variables. El lugar de residencia de los alumnos también marca algunas diferencias en los componentes de los instrumentos de resiliencia y depresión.
\end{abstract}

Palabras clave: Resiliencia, depresión, violentos, no violentos, adolescentes, conducta.

\section{ABSTRACT \\ RESILIENCE AND DEPRESSION IN HIGH SCHOOL STUDENTS METROPOLITAN LIMA WITH OR WITHOUT PARTICIPATING IN VIOLENT ACTS}

A correlational study was conducted between resilience and depression in educational institutions of Lima, with male and female students from 3rd-5fth secondary, the which a party not involved in violence and other self. The instruments used for assessment were the Resilience Scale and Adolescent Depression Inventory. Results show that positive correlations exist on internal components of both tests, while the correlation between them the relationship is more diverse. We identified differences between violent and nonviolent students. as to the confidence, authoritarianism and suicidal thoughts factors. Also, in comparison intersex, identified differences between women and men regarding the degree of emotional control, decision making, self-criticism, tendency to weep, and other variables. The place of students residence also mark some differences in components of resilience and depression instruments.

Keywords: Resilience, depression, violent, non violent, teens, behavior.

1 Docente Principal de la Facultad de Psicología de la UNMSM. E-maill: cvelasquezc@unmsm.edu.pe 
De acuerdo con el Plan Nacional de Salud Mental, publicado por el Ministerio de Salud del Perú en 2005, no hay una información consistente y organizada sobre las epidemiologías depresiva y psicopática en nuestro país, pero presenta algunos datos sintomáticos: en 1997 se identificó la prevalencia de un 32.6\% de depresión en un solo distrito de la capital, considerando sus efectos sobre la población femenina y masculina en períodos del desarrollo particularmente sensibles (respectivamente climaterio y temprana juventud). Posteriormente, en 2002, el Instituto Honorio Delgado-Hideyo Noguchi señaló en su estudio epidemiológico metropolitano un $19 \%$ general de prevalencia depresiva $(14.5 \%$ masculino y $23.3 \%$ femenino).

En el mismo documento también se reportan datos en cuanto a la permisividad frente a la psicopatía o tolerancia hacia conductas delictivas, entendida en términos de mentira frecuente, violencia y robo. La población adulta muestra un nivel de permisividad del $11.6 \%$, y tendencias psicopáticas presentes en el $4.7 \%$ de la población. Esos datos varían en la población adolescente, donde la permisividad es menor que en los adultos (9.9\%) pero las tendencias psicopáticas son superiores (39.4\%); tanto en Lima como en el Callao. Asimismo, la prevalencia de tendencias violentas es patente en el $30.2 \%$ de la población encuestada (18-91 años), comprendiendo problemas de abuso físico a menores y peleas con armas. Por cierto que en las encuestas se trasluce la falta de amor y ternura de los padres como algo que afecta a individuos con trastornos de ansiedad $(29.1 \%)$ y depresión (24\%) (MINSA, 2005).

Así, la relación entre depresión y tendencias psicopáticas antisociales que llevan a la violencia parece encontrarse en la "autodestructividad" experimentada por el individuo violento frente a la desesperanza que siente por sus propias limitaciones y las del entorno. Como se dice en la fuente de información que estamos utilizando, con esto "tenemos un indicador que nos obliga a intervenir a favor de la infancia y adolescencia como prioridad en la promoción y prevención de salud mental" (MINSA, 2005, p. 10). Es de suponer que la prevención conductual y cognitiva de esa patología en la población afectada pase por fomentar la capacidad de afrontamiento, o sea, la resiliencia (Lamas y Lara, 2002; Invar, 2007; Quintana, Montgomery y Malaver, 2009).

Teniendo en cuenta lo dicho, los objetivos de la presente investigación pretenden esclarecer, en primer lugar, si hay relación entre la resiliencia y la depresión en la muestra estudiada, $y$, en segundo lugar, si es que existen diferencias en la resiliencia y la depresión en los alumnos con y sin participación en actos violentos en función a la edad, el sexo, el grado de instrucción y el lugar de residencia.

\section{Variables bajo estudio}

\section{Resiliencia}

Para introducir el concepto de resiliencia frente a las frustraciones junto con la capacidad de adaptación y la persistencia frente a la adversidad que muestran ciertas personas, Rutter aplicó una metáfora física extraída del concepto de capacidad de los edificios bien 
construidos para soportar presiones sin deformarse. En términos comportamentales, pues, la resiliencia comprende competencias individuales de enfrentamiento y fortalecimiento a través de las experiencias adversas, incluso traumáticas, que se sufren (Henderson, 2002; Vera, Carbelo y Vecina, 2006).

Las características de la resiliencia no están relacionadas necesariamente con la capacidad intelectual ni con la acomodación socioeconómica, mas sí con factores de calidad de vida y de salud mental (Vsillant y Davis, 2000). Mediante su análisis se procuran esclarecer las razones por las cuales sujetos de edad diversa sobreviven y progresan en ambientes de riesgo, donde predominan la pobreza, la violencia intrafamiliar, las patologías familiares o los desastres naturales. Se busca esencialmente reproducir los mecanismos de adaptación implicados, para la promoción social del comportamiento adaptativo llamado resiliente (Infante, 2002; Salgado, 2005; Flores, 2007, Bulnes, et al, 2008). En cierto sentido, como dice Quintero (2005), la resiliencia constituye un cambio de paradigma al centrar el enfoque no en las debilidades, sino en las fortalezas del individuo.

\section{Depresión}

La revisión histórica de Jackson (1986/1989) acerca de la antiguamente llamada melancolía, señala al médico británico Richard Blackmoer como el autor del término depresión en el siglo XVIII, para caracterizar un cuadro clínico de prevalencia de aproximadamente $3 \%$ en la población general de los países occidentales. Según el Manual Diagnóstico y Estadístico de los Trastornos Mentales (DSM-IV), la depresión incluye síntomas de disforia y pérdida de interés y placer en casi toda actividad, presentándose de manera persistente y con cambios somáticos, afectivos y cognitivos muy acusados, tales como la pérdida de peso, la astenia, el insomnio. La baja autoestima y la ideación suicida (Asociación Psiquiátrica Americana, 1994/2002). Su origen puede tener, de acuerdo con cada caso particular, raíces biológicas (incluso genéticas) o psicosociales.

Partiendo de un punto de vista conductual, la conceptualización de las características psicológicas de esta disforia se puede abordar desde los modelos cognitivo-conductual de Beck e integrador de Staats.

Beck (1995/2000; Beck, Rush, Shaw, y Emery, 1979/1983) considera que en la aparición de perturbaciones emocionales juega un papel decisivo la distorsión del procesamiento de la información: los individuos tienden a percibir negativamente el ambiente y los acontecimientos del contexto, abstrayendo selectivamente, sobregeneralizando, dicotomizando, maximizando o minimizando ciertos aspectos. Eso incluye las creencias y pensamientos, y también los estados anímicos ya mencionados que los acompañan.

Por su parte, Staats (1996/1997), Carrillo, Collado, Rojo y Staats (2006) hace hincapié sobre la naturaleza interactiva del repertorio emotivo-motivacional del individuo: los estímulos emocionales de su vida, según su índole positiva o negativa, le provocan conductas de aproximación o de escape, evitación u otras. Cuando alguien se expone a múltiples fuentes de estimulación emocional, las emociones se suman, siendo el resultado de una intensidad mayor a la experimentada con una sola respuesta emocional negativa, de tal forma que produce un trastorno. Este estado a su vez evoca respuestas en otros 
repertorios del sistema total de personalidad, como el lingüístico-cognoscitivo (p. ej. pensamientos y verbalizaciones depresivas recurrentes), y el sensorial-motor (p. ej. bajas tasas de actividad), además de repercutir sobre las demás respuestas del propio repertorio emotivo-motivacional (p. ej. irritación o tristeza constantes ante sucesos inocuos).

\section{MÉTODO}

\section{Muestra}

Se utilizó un diseño descriptivo correlacional y comparativo. El estudio se realizó en 29 instituciones educativas de los Conos Norte, Este y Sur, de Lima Cercado y Callao, con una muestra de 1309 alumnos, de los cuales 641 están clasificados como que no participan y 668 como que participan en actos violentos, del tercero al quinto de secundaria, de género masculino y femenino, con una edad promedio de 16 años. Para la obtención de la muestra, se aplicó el procedimiento de muestreo probabilístico.

\section{Instrumentos}

Escala de Resiliencia de Wagnild y Young (1993), originalmente con 25 reactivos en grado de aprobación o desaprobación en una escala de 7 puntos, respecto a cinco subtests o factores: Confianza en sí mismo, ecuanimidad, satisfacción, sentirse bien solo y perseverancia. Al no cumplir inicialmente con los criterios de validez y confiabilidad, fue sometida a un análisis factorial, quedando finalmente reestructurada en cuatro componentes: confianza en sí mismo, control interno, perseverancia y optimismo.

Inventario de Depresión en la Adolescencia (IDA), con 40 reactivos cuya puntuación va del 1 al 5 desde "nunca" a "siempre". El inventario, adaptado por Aranda (1999), incluye las cuatro categorías consideradas por Beck. Al ser sometido a los criterios de validez y construcción se eliminaron algunos ítems, pero al final mantuvo su misma estructura: cognitiva, emocional, motivacional y neurovegetativa.

\section{RESULTADOS}

En la Tabla N. ${ }^{\circ} 1$ se puede apreciar que hay una mayor cantidad de relaciones inversas entre las áreas de resiliencia y depresión. A fin de determinar el tipo de estadístico inferencial a ser utilizado, se aplicó la prueba de normalidad a cada una de las variables obteniéndose evidencias de que debe optarse por un análisis no paramétrico al grado de $p 0.01$ (Tabla N. ${ }^{\circ} 2$ ). 
Tabla N. ${ }^{0}$ 1. Relación entre las áreas de resilencia y depresión.

\begin{tabular}{|c|c|c|c|c|c|c|c|c|}
\hline Factores & csm & cem & per & opt & $\operatorname{cog}$ & emo & mot & neve \\
\hline Confianza en sí mismo (csm) & $\begin{array}{r}1.00 \\
1309\end{array}$ & $\begin{array}{c}, 380^{* *} \\
, 000 \\
1308\end{array}$ & $\begin{array}{l}, 504^{* * *} \\
, 000 \\
1308\end{array}$ & $\begin{array}{c}467^{\text {** }} \\
000 \\
1306\end{array}$ & $\begin{array}{c}-, 342^{* *} \\
, 000 \\
1309\end{array}$ & $\begin{array}{c}-, 188^{* *} \\
, 000 \\
1308\end{array}$ & $\begin{array}{c}-, 300^{* *} \\
, 000 \\
1308\end{array}$ & $\begin{array}{c}-, 094^{* *} \\
, 001 \\
1309\end{array}$ \\
\hline Control emocional (cem) & & $\begin{array}{l}1.00 \\
1308\end{array}$ & $\begin{array}{l}, 325^{* *} \\
, 000 \\
1307\end{array}$ & $\begin{array}{c}, 441^{\text {*** }} \\
, 000 \\
1305\end{array}$ & $\begin{array}{c}-, 012 \\
, 669 \\
1308\end{array}$ & $\begin{array}{l}, 057^{*} \\
, 039 \\
1307\end{array}$ & $\begin{array}{l}, 026 \\
, 354 \\
1307\end{array}$ & $\begin{array}{c}, 079^{* *} \\
, 004 \\
1308\end{array}$ \\
\hline Perseverancia (per) & & & $\begin{array}{l}1.00 \\
1308\end{array}$ & $\begin{array}{c}, 394^{* * *} \\
, 000 \\
1305\end{array}$ & $\begin{array}{c}-, 160^{* *} \\
, 000 \\
1308\end{array}$ & $\begin{array}{c}-, 091^{* *} \\
, 001 \\
1307\end{array}$ & $\begin{array}{c}-, 138^{* * *} \\
, 000 \\
1307\end{array}$ & $\begin{array}{r}-, 049 \\
, 077 \\
1308\end{array}$ \\
\hline Optimismo (opt) & & & & $\begin{array}{l}1.000 \\
1306\end{array}$ & $\begin{array}{r}-, 031 \\
, 264 \\
1306\end{array}$ & $\begin{array}{l}, 036 \\
, 189 \\
1305\end{array}$ & $\begin{array}{c}-, 029 \\
, 301 \\
1305\end{array}$ & $\begin{array}{l}, 004 \\
, 881 \\
1306\end{array}$ \\
\hline Cognitivos (cog) & & & & & $\begin{array}{c}1,000 \\
1309\end{array}$ & $\begin{array}{l}, 690^{* *} \\
, 000 \\
1308\end{array}$ & $\begin{array}{c}, 634^{* *} \\
, 000 \\
1308\end{array}$ & $\begin{array}{c}, 362^{* *} \\
, 000 \\
1309\end{array}$ \\
\hline Emocionales (emo) & & & & & & $\begin{array}{c}1,000 \\
1308\end{array}$ & $\begin{array}{c}, 533^{* *} \\
, 000 \\
1307\end{array}$ & $\begin{array}{c}, 352^{* *} \\
, 000 \\
1308\end{array}$ \\
\hline Motivacionales (mot) & & & & & & & $\begin{array}{c}1,000 \\
1308\end{array}$ & $\begin{array}{c}, 302^{* *} \\
, 000 \\
1308\end{array}$ \\
\hline Neurovegetativos (neve) & & & & & & & & $\begin{array}{c}1,000 \\
1309\end{array}$ \\
\hline
\end{tabular}

** Correlación significativa al 0.02 .

* Correlación significativa al 0.05 .

Tabla N. ${ }^{\circ}$ 2. Prueba de Kolmogorov-Smirnov.

\begin{tabular}{lccccc}
\hline Factores & N & \multicolumn{2}{c}{ Parámetros normales } & $\begin{array}{c}\text { Z de Kolmogorov- } \\
\text { Smirnov }\end{array}$ & $\begin{array}{c}\text { Sig-asintót. } \\
\text { (bilateral) }\end{array}$ \\
\hline \multicolumn{5}{c}{ Media } & Desv. típica \\
\hline Confianza en sí mismo & 1309 & 47,9274 & 8,17956 & 2,841 &, 000 \\
control emocional & 1308 & 28,3135 & 5,46054 & 2,390 &, 000 \\
Perseverancia & 1308 & 20,6529 & 3,94786 & 2,921 &, 000 \\
Optimismo & 1306 & 29,3767 & 4,96336 & 2,255 &, 000 \\
Cognitivos & 1309 & 39,2147 & 13,86679 & 3,533 &, 000 \\
Emocionales & 1308 & 19,9610 & 5,26054 & 2,920 &, 000 \\
Motivacionales & 1308 & 19,9602 & 5,23743 & 2,270 &, 000 \\
Neurovegetativos & 1309 & 4,6241 & 2,16750 & 5,385 &, 000 \\
\hline
\end{tabular}

Por otro lado, en la Tabla N. 3 puede apreciarse que sólo existen diferencias significativas en el área de confianza en sí mismo de la resiliencia y las áreas cognitiva y motivacional de la depresión. 
Tabla N. ${ }^{0}$ 3. Comparación entre las áreas de resiliencia y depresión con la condición de alumnos violentos y no violentos.

\begin{tabular}{|c|c|c|c|c|}
\hline Factores & Condición de Ss & $\mathbf{N}$ & Rango prom. & Suma de rangos \\
\hline \multirow{2}{*}{ Confianza en sí mismo } & No violentos & 641 & 694,09 & 444912,50 \\
\hline & Violentos & 668 & 617,49 & 412482,50 \\
\hline \multirow{2}{*}{ Control emocional } & No violentos & 640 & 666,77 & 426730,50 \\
\hline & Violentos & 668 & 642,75 & 429355,50 \\
\hline \multirow{2}{*}{ Perseverancia } & No violentos & 640 & 667,36 & 427113,00 \\
\hline & Violentos & 668 & 642,18 & 428073,00 \\
\hline \multirow{2}{*}{ Optimismo } & No violentos & 638 & 664,31 & 423830,00 \\
\hline & Violentos & 668 & 643,18 & 429641,00 \\
\hline \multirow{2}{*}{ Cognitivos } & No violentos & 641 & 602,93 & 386475,00 \\
\hline & Violentos & 668 & 704,97 & 470920,00 \\
\hline \multirow{2}{*}{ Emocionales } & No violentos & 640 & 658,48 & 421424,50 \\
\hline & Violentos & 668 & 650,69 & 434661,50 \\
\hline \multirow{2}{*}{ Motivacionales } & No violentos & 640 & 569,20 & 364287,50 \\
\hline & Violentos & 668 & 736,23 & 491798,50 \\
\hline \multirow{2}{*}{ Neurovegetativos } & No violentos & 641 & 660,80 & 423571,00 \\
\hline & Violentos & 668 & 649,44 & 433824,00 \\
\hline Factores & $\begin{array}{c}\text { U de } \\
\text { Mann-Whitney }\end{array}$ & $\begin{array}{c}\text { W de } \\
\text { Wilcoxon }\end{array}$ & $\mathbf{Z}$ & $\begin{array}{l}\text { Sig. asirtòt } \\
\text { (bilateral) }\end{array}$ \\
\hline Confianza en sí mismo & 189036,500 & 412482,500 & $-3,668$ &, 000 \\
\hline Control emocional & 205909,500 & 429355,500 & $-1,152$ &, 249 \\
\hline Perseverancia & 205527,000 & 428973,000 & $-1,209$ &, 227 \\
\hline Optimismo & 206195,000 & 429641,000 & $-1,014$ &, 310 \\
\hline Cognitivos & 180714,000 & 386475,000 & $-4,884$ &, 000 \\
\hline Emocionales & 211215,500 & 434661,500 &,- 373 & ,709 \\
\hline Motivacionales & 159167,500 & 364287,500 & $-8,008$ &, 000 \\
\hline Neurovegetativos & 210378,000 & 433824,000 &,- 550 & ,582 \\
\hline
\end{tabular}

A su vez, en la Tabla N. ${ }^{\circ} 4$ se observa que existen diferencias significativas en las áreas de perseverancia y optimismo de la resiliencia y el factor motivacional de la depresión. 
Tabla N. ${ }^{\circ}$ 4. Comparación entre las áreas de la resiliencia y la depresión con el sexo.

\begin{tabular}{|c|c|c|c|c|}
\hline Factores & Sexo & $\mathbf{N}$ & Mean Rank & Sum of Ranks \\
\hline \multirow{2}{*}{ Confianza en sí mismo } & Masculino & 870 & 638,80 & 555753,50 \\
\hline & Femenino & 439 & 687,11 & 301641,50 \\
\hline \multirow{2}{*}{ Control emocional } & Masculino & 870 & 639,44 & 556308,50 \\
\hline & Femenino & 438 & 684,42 & 299777,50 \\
\hline \multirow{2}{*}{ Perseverancia } & Masculino & 869 & 645,14 & 560630,00 \\
\hline & Femenino & 439 & 673,02 & 295456,00 \\
\hline \multirow{2}{*}{ Optimismo } & Masculino & 868 & 644,24 & 559303,00 \\
\hline & Femenino & 438 & 671,84 & 294268,00 \\
\hline \multirow{2}{*}{ Cognitivos } & Masculino & 870 & 621,36 & 540500,00 \\
\hline & Femenino & 439 & 721,86 & 316895,00 \\
\hline \multirow{2}{*}{ Emocionales } & Masculino & 870 & 576,42 & 501484,00 \\
\hline & Femenino & 438 & 809,59 & 354602,00 \\
\hline \multirow{2}{*}{ Motivacionales } & Masculino & 869 & 648,25 & 563331,50 \\
\hline & Femenino & 439 & 666,87 & 292754,50 \\
\hline \multirow{2}{*}{ Neurovegetativos } & Masculino & 870 & 600,41 & 522354,03 \\
\hline & Femenino & 439 & 763,19 & 335041,00 \\
\hline Factores & Mann-Whitney & Wilcoxon W & $\mathbf{Z}$ & Asymp. Sig. \\
\hline Confianza en sí mismo & 176868,500 & 555753,500 & $-2,185$ & 029 \\
\hline control emocional & 177423,500 & 556308,500 & $-2,086$ &, 042 \\
\hline Perseverancia & 183615,000 & 560630,000 & $-1,364$ & ,306 \\
\hline Optimismo & 183057,000 & 559203,000 & $-1,251$ &, 211 \\
\hline Cognitivos & 161615,000 & 540500,000 & $-4,547$ &, 000 \\
\hline Emocionales & 122999,000 & 501484,000 & $-10,556$ &, 000 \\
\hline Motivacionales & 185316,500 & 563331,500 &,- 843 & ,399 \\
\hline Neurovegetativos & 143469,000 & 522354,000 & $-7,447$ &, 000 \\
\hline
\end{tabular}

En la Tabla N. ${ }^{\circ} 5$, el grado de instrucción presenta diferencias significativas sólo en el área de control emocional, y en la Tabla N.$^{\circ} 6$ no se notan diferencias significativas entre las áreas de resiliencia y depresión. 
Tabla N. ${ }^{0}$ 5. Comparación entre las áreas de resiliencia y depresión con el grado de instrucción.

\begin{tabular}{|c|c|c|c|}
\hline Factores & Grado de instrucción & $\mathbf{N}$ & Mean Rant \\
\hline Confianza en sí mismo & $\begin{array}{l}\text { Tercero de secundaria } \\
\text { Cuarto de secundaria } \\
\text { Quinto de secundaria }\end{array}$ & $\begin{array}{l}414 \\
455 \\
440\end{array}$ & $\begin{array}{l}555753,50 \\
301641,50\end{array}$ \\
\hline Control emocional & $\begin{array}{l}\text { Tercero de secundaria } \\
\text { Cuarto de secundaria } \\
\text { Quinto de secundaria }\end{array}$ & $\begin{array}{l}413 \\
455 \\
440\end{array}$ & $\begin{array}{c}556308,500 \\
299777,50\end{array}$ \\
\hline Perseverancia & $\begin{array}{l}\text { Tercero de secundaria } \\
\text { Cuarto de secundaria } \\
\text { Quinto de secundaria }\end{array}$ & $\begin{array}{l}414 \\
455 \\
439\end{array}$ & $\begin{array}{l}560630,00 \\
295456,00\end{array}$ \\
\hline Optimismo & $\begin{array}{l}\text { Tercero de secundaria } \\
\text { Cuarto de secundaria } \\
\text { Quinto de secundaria }\end{array}$ & $\begin{array}{l}414 \\
455 \\
439\end{array}$ & $\begin{array}{l}559303,00 \\
294368,00\end{array}$ \\
\hline Cognitivos & $\begin{array}{l}\text { Tercero de secundaria } \\
\text { Cuarto de secundaria } \\
\text { Quinto de secundaria }\end{array}$ & $\begin{array}{l}414 \\
455 \\
440\end{array}$ & $\begin{array}{l}540500,00 \\
316895,00\end{array}$ \\
\hline Emocionales & $\begin{array}{l}\text { Tercero de secundaria } \\
\text { Cuarto de secundaria } \\
\text { Quinto de secundaria }\end{array}$ & $\begin{array}{l}414 \\
454 \\
440\end{array}$ & $\begin{array}{l}501484,00 \\
354602,00\end{array}$ \\
\hline Motivacionales & $\begin{array}{l}\text { Tercero de secundaria } \\
\text { Cuarto de secundaria } \\
\text { Quinto de secundaria }\end{array}$ & $\begin{array}{l}414 \\
455 \\
439\end{array}$ & $\begin{array}{l}563331,50 \\
292754,50\end{array}$ \\
\hline Neurovegetativos & $\begin{array}{l}\text { Tercero de secundaria } \\
\text { Cuarto de secundaria } \\
\text { Quinto de secundaria }\end{array}$ & $\begin{array}{l}414 \\
455 \\
440\end{array}$ & $\begin{array}{l}522354,03 \\
335041,00\end{array}$ \\
\hline & $\mathrm{Chi}^{2}$ & dt & Asymp. Sig. \\
\hline Confianza en sí mismo & 3,368 & 2 &, 186 \\
\hline Control emocional & 9,307 & 2 &, 010 \\
\hline Perseverancia & 1,623 & 2 & ,444 \\
\hline Optimismo & 1,328 & 2 &, 515 \\
\hline Cognitivos & 3,381 & 2 &, 184 \\
\hline Emocionales & 4,360 & 2 &, 113 \\
\hline Motivacionales & 2,336 & 2 & ,311 \\
\hline Neurovegetativos & ,705 & 2 & ,703 \\
\hline
\end{tabular}


Tabla N. ${ }^{0}$ 6. Comparación entre las áreas de resiliencia y depresión con la edad.

\begin{tabular}{|c|c|c|c|c|}
\hline Factores & Sexo & $\mathbf{N}$ & Mean Rank & Suma de rangos \\
\hline \multirow{2}{*}{ Confianza en sí mismo } & Adolescentes & 966 & 648,78 & 636724,50 \\
\hline & Jóvenes & 343 & 672,51 & 230670,50 \\
\hline \multirow{2}{*}{ Control emocional } & Adolescentes & 965 & 642,87 & 630372,00 \\
\hline & Jóvenes & 343 & 687,21 & 235714,00 \\
\hline \multirow{2}{*}{ Perseverancia } & Adolescentes & 966 & 661,54 & 639049,00 \\
\hline & Jóvenes & 342 & 634,61 & 217036,98 \\
\hline \multirow{2}{*}{ Optimismo } & Adolescentes & 966 & 652,07 & 629895,00 \\
\hline & Jóvenes & 340 & 657,58 & 223576,00 \\
\hline \multirow{2}{*}{ Cognitivos } & Adolescentes & 966 & 647,32 & 625306,50 \\
\hline & Jóvenes & 343 & 676,64 & 233088,52 \\
\hline \multirow{2}{*}{ Emocionales } & Adolescentes & 965 & 653,81 & 630927,00 \\
\hline & Jóvenes & 343 & 656,44 & 225159,00 \\
\hline \multirow{2}{*}{ Motivacionales } & Adolescentes & 966 & 652,61 & 630418,00 \\
\hline & Jóvenes & 342 & 659,85 & 225668,00 \\
\hline \multirow{2}{*}{ Neurovegetativos } & Adolescentes & 966 & 649,43 & 627353,00 \\
\hline & Jóvenes & 343 & 670,68 & 230042,00 \\
\hline Factores & Mann-Whitney & Wilcoxon W & $\mathbf{Z}$ & Asymp. Sig. \\
\hline Confianza en sí mismo & 159663,500 & 626724,500 & -.999 & ,318 \\
\hline Control emocional & 154277.000 & 620372,000 & $-1,871$ &, 061 \\
\hline Perseverancia & 158184,000 & 217037,000 & $-1,136$ & ,256 \\
\hline Optimismo & 163834,000 & 629891,000 &,- 232 & ,816 \\
\hline Cognitivos & 158245,500 & 635306,500 & $-1,235$ &, 217 \\
\hline Emocionales & 164832,000 & 630927,000 &,- 111 & ,911 \\
\hline Motivacionales & 163357,000 & 630418,000 & -.305 & ,760 \\
\hline Neurovegetativos & 160292,000 & 627313,000 & -.901 & ,365 \\
\hline
\end{tabular}

En cambio, la Tabla N. ${ }^{o} 7$ muestra que sí existen diferencias significativas entre los Conos en las áreas control de sí mismo, perseverancia y optimismo de la depresión y el área cognitiva de la depresión. 
Tabla N. ${ }^{0}$ 7. Comparación entre las áreas de resiliencia y depresión según el cono de residencia.

\begin{tabular}{|c|c|c|c|}
\hline Factores & Cono & $\mathbf{N}$ & Megin $\mathrm{g}$ \\
\hline \multirow{5}{*}{ Confianza en sí mismo } & Cono Norte & 216 & 560,97 \\
\hline & Cono Este & 266 & 681,07 \\
\hline & Cono Sur & 374 & 713,60 \\
\hline & Cono Lima Cercado & 309 & 598,68 \\
\hline & Cono Callao & 144 & 716,56 \\
\hline \multirow{5}{*}{ Control emocional } & Cono Norte & 216 & 634,78 \\
\hline & Cono Este & 265 & 664,52 \\
\hline & Cono Sur & 374 & 671,51 \\
\hline & Cono Lima Cercado & 309 & 621,24 \\
\hline & Cono Callao & 144 & 692,84 \\
\hline \multirow{5}{*}{ Perseverancia } & Cono Norte & 216 & 588,61 \\
\hline & Cono Este & 265 & 669,12 \\
\hline & Cono Sur & 374 & 688,74 \\
\hline & Cono Lima Cercado & 309 & 641,20 \\
\hline & Cono Callao & 144 & 666,04 \\
\hline \multirow{5}{*}{ Optimismo } & Cono Norte & 216 & 595,72 \\
\hline & Cono Este & 263 & 675,06 \\
\hline & Cono Sur & 374 & 698,89 \\
\hline & Cono Lima Cercado & 309 & 603,89 \\
\hline & Cono Callao & 144 & 689,38 \\
\hline \multirow{5}{*}{ Cognitivos } & Cono Norte & 216 & 676,24 \\
\hline & Cono Este & 266 & 698,94 \\
\hline & Cono Sur & 374 & 645,97 \\
\hline & Cono Lima Cercado & 309 & 648,79 \\
\hline & Cono Callao & 144 & 578,75 \\
\hline \multirow{5}{*}{ Emocionales } & Cono Norte & 216 & 650,11 \\
\hline & Cono Este & 266 & 697,24 \\
\hline & Cono Sur & 374 & 671,72 \\
\hline & Cono Lima Cercado & 308 & 614,23 \\
\hline & Cono Callao & 144 & 623,56 \\
\hline \multirow{5}{*}{ Motivacionales } & Cono Norte & 216 & 711,50 \\
\hline & Cono Este & 266 & 652,47 \\
\hline & Cono Sur & 374 & 621,57 \\
\hline & Cono Lima Cercado & 308 & 651,62 \\
\hline & Cono Callao & 144 & 664,45 \\
\hline \multirow{5}{*}{ Neurovegetativos } & Cono Norte & 216 & 650,04 \\
\hline & Cono Este & 266 & 698,88 \\
\hline & Cono Sur & 374 & 650,84 \\
\hline & Cono Lima Cercado & 309 & 640,11 \\
\hline & Cono Callao & 144 & 624,14 \\
\hline Factores & $\mathrm{Chi}^{2}$ & dt & Asymp. Sig. \\
\hline Confianza en si mismo & 34,349 & 4 &, 000 \\
\hline Control emocional & 5,432 & 4 & ,246 \\
\hline Perseverancia & 10,623 & 4 &, 061 \\
\hline Optimismo & 18,067 & 4 &, 001 \\
\hline Cognitivos & 10,438 & 4 &, 034 \\
\hline Emocionales & 8,711 & 4 & 069 \\
\hline Motivacionales & 7,914 & 4 & ,095 \\
\hline Neurovegetativos & 5,233 & 4 &, 064 \\
\hline
\end{tabular}




\section{DISCUSIÓN}

En resumen, con respecto a la relación entre los componentes de las escalas de resiliencia y depresión existen correlaciones positivas, entre bajas, moderadas y significativas. $\mathrm{Su}$ relación intracomponentes también es baja, significativa, y en el $47 \%$ de los casos expresan relaciones inversas, así como en el $12 \%$ directa.

En cuanto al factor confianza en sí mismo de la escala de resiliencia, este es mayor en los sujetos no violentos en comparación con los sujetos violentos, mientras que en los componentes cognitivos y motivacionales del inventario de depresión es mayor en los individuos violentos en comparación con los neurovegetativos. Los sujetos violentos son autocráticos y presentan ideas suicidas, en comparación con los no violentos, lo que apoya las proposiciones de Carrillo, Collado, Rojo, y Staats (2006) en relación con el poder del principio de la adición de las emociones, en este caso negativas. Las mujeres, en comparación con los varones, se caracterizan por tener un mejor control emocional, pero también por ser indecisas, autocríticas, tener propensión al llanto, perder la alegría e interés por las cosas, así como el apetito, y sufrir de insomnio.

No se ve que el grado de instrucción y la edad influyan en ninguna de las escalas, lo que coincide con resultados de reportes anteriores sobre resiliencia (Bulnes, et al, 2008). Al parecer, la educación no juega mayor papel que el contexto afectivo de la familia en cuanto a la formación de comportamiento resiliente. Sin embargo, sí es hasta cierto punto sorprendente la falta de relación entre la educación y la depresión -en el estudio de Aranda (1999) se halló una relación inversa-, dada la presunción generalmente aceptada de que a mejor rendimiento académico suele haber menor tendencia depresiva y viceversa.

En cambio, sí existen diferencias significativas en cuanto al lugar de residencia de los individuos en los componentes confianza en sí mismo, perseverancia y optimismo de la resiliencia, así como también en el componente cognitivo del inventario de depresión, lo que sin duda debe constituir un aspecto a investigar en el futuro.

Los instrumentos, debidamente adaptados a nuestra realidad, logran perfilar a los alumnos violentos como no violentos, de acuerdo al sexo y el lugar de residencia. De acuerdo con el estudio, estas pruebas se encuentran como válidos y confiables.

\section{NOTA DE RECONOCIMIENTO}

El responsable reconoce la participación a los siguientes miembros del equipo de investigación que cmoparten la autoría del siguiente estudio de investigación: Lupe García A., Gilmar Guevara, José Vega y Pedro García; así como la de los colaboradores Ricardo Pomalaya, Gianina Lobatón, Davis Durand, David Jáuregui y Betty Peña.

\section{REFERENCIAS BIBLIOGRÁFICAS}

1. Aranda, M. (1999). Construcción de un inventario para evaluar la depresión en adolescentes. Tesis de Magíster. México: UNAM.

2. Asociación Psiquiátrica Americana (1994/2002). DSM-IV. Manual diagnóstico y estadístico de los trastornos mentales. Barcelona: Masson. Beck, 
3. Beck, A. Rush, J., Shaw, B. y Emery, G. (1979/1983). Terapia cognitiva de la depresión. Bilbao: Descleé de Brouwer.

4. Beck, J. (1995/2000). Terapia cognitiva. Conceptos básicos y profundización. Barcelona: Gedisa.

5. Bulnes, M., Ponce, C., Huerta R., Álvarez C., Santibáñez, W., Atalaya, M., Aliaga, J. y Morocho, J. (2008). Resiliencia y estilos de socialización parental en escolares de 4to y 5to año de secundaria de Lima Metropolitana. Revista de Investigación en Psicología de la Universidad Nacional Mayor de San Marcos, 11(2), 67-91.

6. Carrillo, J.M.; Collado, S.; Rojo, N. y Staats, A. W. (2006). El papel de las emociones positivas y negativas en la predicción de depresión: el principio de adición de las emociones en el Conductismo Psicológico. Clínica y Salud, 17(3), 277-295.

7. Flores, J. J. (2007). Resiliencia en familias víctimas de violencia política en Ayacucho. Revista Peruana de Psicología, 1(1), 5-32.

8. Henderson, E. (2002). Nuevas tendencias en resiliencia. En A. Melillo y E. Suarez (Dirs.). Resiliencia: Descubriendo las propias fortalezas (pp. 19-40). Buenos Aires: Paidós.

9. Infante, F. (2002). La resiliencia como proceso: Una revisión de la literatura reciente. En A. Melillo y E. Suárez (Dirs.). Resiliencia: Descubriendo las propias fortalezas (pp. 41-53). Buenos Aires: Paidós.

10. Invar, J. (2007). Modelo integrativo enfocado en las soluciones para el desarrollo de la resiliencia individual y familiar. Perspectivas sistémicas: La Nueva Comunicación, $N^{o}$ 94-95. Disponible en: http://www.redsistemica.com.ar/articulo94-4.htm

11. Jackson, S. W. (1986/1989). Historia de la melancolía y la depresión. Desde los tiempos hipocráticos a la época moderna. Madrid: Turner.

12. Lamas, H y Lara, M (2002). Resiliencia: optimización de las potencialidades. Docencia, II(4), 29-31.

13. MINSA (2005). Plan Nacional de Salud Mental. Disponible en: http://www.minsa. gob.pe/portal/p2005/documentos/cns/PlanNacionalSaludMental-Set2005.doc

14. Quintana, A., Montgomery, W. y Malaver, C. (2009). Modos de afrontamiento y conducta resiliente en adolescentes espectadores de violencia entre pares. Revista de Investigación en Psicología de la Universidad Nacional Mayor de San Marcos, 12(1), 153-171.

15. Quintero, A. M. (2005). Resiliencia: Contexto no clínico para trabajo social. Revista Latinoamericana de Ciencias Sociales, Niñez y Juventud, 3, 2-16.

16. Salgado, A. C. (2005). La ciencia y su respuesta frente a la adversidad: Estudios desde la perspectiva de la resiliencia. Cultura, XXIII(19), 393-417.

17. Staats, A. W. (1996/1997). Conducta y personalidad: Conductismo psicológico. Bilbao: Descleé de Brouwer. 
18. Vera, B.; Carbelo, B. y Vecina, M. L. (2006). La experiencia traumática desde la psicología positiva: resiliencia y crecimiento postraumático. Papeles del Psicólogo, 27(1), 40-49.

19. Vsillant, G. y Davis, T. (2000). Social-emotional intelligence and midlife: Resilience in schoolboys with low tested intelligence. American Journal of Orthopsychiatry, 70(2), 215-222.

20. Wagnild,G. \& Young, H. (1993). Development and psycometric evaluation of the Resilience Scale. Journal of Nursing Measurement 1(2), 165-177. 